\title{
Un polo textil impulsado desde la periferia. Abordajes sobre La Salada a partir de investigaciones en Ciencias Sociales
}

\author{
A textile pole driven from the periphery: approaches on La Salada \\ based on research in Social Sciences \\ Juan Martín Bello jmbello@live.com.ar \\ https://orcid.org/0000-0001-5653-3461 \\ Instituto de Investigaciones Gino Germani; Facultad de Ciencias Sociales; Universidad de \\ Buenos Aires (Argentina)
}

\section{Resumen}

En el presente artículo se realiza una revisión bibliográfica de las investigaciones académicas que abordaron el fenómeno de la feria de La Salada. Para ello se han considerado aquellos 
trabajos que se focalizaron en este conjunto de ferias, ya fuese como su principal objeto de estudio o como un caso relevante dentro de un abordaje más amplio.

A lo largo de las siguientes páginas se expondrán los orígenes de la feria de La Salada, su relevancia para las ciencias sociales, y los modos en que distintos estudios abordaron el fenómeno. En este proceso, se distinguen dos grandes grupos de trabajos, identificando, por un lado, investigaciones centradas en una distinción antinómica entre lo formal y lo informal, y, por otro, estudios que abordan a estas ferias a partir de su dimensión productiva, esto es, a partir de las relaciones y prácticas sociales que consideran específicas del fenómeno.

En el último apartado, se señalan los aportes de estos trabajos y algunas áreas de vacancia que motivan un abordaje de mayor profundidad, vinculadas con los distintos niveles de regularización formal que presentan los locales, el surgimiento de marcas propias y el acercamiento de nuevos públicos.

Palabras clave: Informalidad; La Salada; revisión bibliográfica; sociología de la cultura; sociología del trabajo.

\section{Abstract}

The present article offers a bibliographic review of the academic research on the phenomenon of 'La Salada' market. For this purpose, those works that focused on this conglomerate of markets were identified, either as their principal object of study or as a relevant case within a broader approach.

Throughout the following pages the origins of 'La Salada', its relevance for social sciences, and the ways in which different studies addressed the phenomenon will be exposed. In this process, two large groups of works are distinguished. There can be identified, on the one hand, research focused on an antinomic distinction between formality and informality, and, on the other hand, studies that address these markets from their productive dimension, this is, from the social relations and social practices which are considered specific to the phenomenon.

In the last section, the contributions of these works and some areas of vacancy that motivate an approach with greater depth, linked to the different levels of formal regularization presented by 
Juan Martín Bello. Un polo textil impulsado desde la periferia. Abordajes sobre La Salada a partir de investigaciones en Ciencias Sociales

the premises, the emergence of own brands and the approaching of new publics are pointed out.

Keywords: Informality; La Salada; bibliographic review; culture sociology; sociology of work.

La Salada es un complejo ferial ubicado en la localidad bonaerense de Lomas de Zamora (1) (2). Los orígenes de la feria se remontan al año 1991, cuando un grupo de migrantes bolivianos organizados como cooperativa comenzaron a vender ropa y calzado a precios muy inferiores en relación a los que comúnmente podían encontrarse en el mercado (D'Angiolillo et al., 2010; Ossona, 2010a; Schiavo et al., 2016). El complejo comercial tuvo un primer crecimiento durante los años noventa. En este período, su expansión se produjo debido a un aumento de la pobreza en Argentina que implicó una mayor demanda de los bienes a bajos precios que la feria ofrecía. Durante estos años una proporción significativa de los productos comercializados provenían de importaciones. Luego de la crisis económica del 2001 y de la devaluación de la moneda local, los principales proveedores comenzaron a ser talleristas locales que por to general estaban asociados con los puesteros de la feria (3) (Campos, 2008; Pogliaghi, 2008; 2010; Schiavo et al. 2016).

En la actualidad el complejo de La Salada ocupa un territorio de 20 hectáreas de extensión y está conformado por un total de tres grandes ferias: Punta Mogote, Urkupiña y Ocean, además de numerosos paseos de compras de menores dimensiones y puestos instalados en la vía pública. Las tres ferias funcionan en predios bajo cubierta y son consideradas como "semilegales" debido a que sus puestos se encuentran registrados bajo la figura del monotributo (Campos, 2008; Ossona, 2010a) y a raíz de esto presentarían una relativa formalización. Hasta mediados de 2017 (y con algunas interrupciones) funcionó también la feria de La Ribera, ubicada en la vía pública del lado del Riachuelo y en condiciones de menor formalidad (4). Cada una de las tres ferias "legales" consta de alrededor de 2000 puestos de venta. En su conjunto, el complejo comercial de La Salada emplea cerca de 6000 familias y tiene un volumen de compradores que en 2009 posicionó al complejo en el tercer lugar entre los principales shoppings de Argentina (5), con un número de 50.000 visitantes por día de feria 
(Abba, 2009; D’Angiolillo et al., 2010; Gago, 2012) (6). Por otro lado, al interior del país, La Salada abastece a otras 200 ferias de menor envergadura.

Durante los últimos años el fenómeno de La Salada cobró visibilidad en los medios de comunicación y en la investigación académica en ciencias sociales. Esto se debe tanto a su crecimiento económico (que se manifiesta en el volumen de compradores, el número de puestos, la cantidad de personas empleadas y su relación con otros sectores de la economía formal e informal) como también a los rasgos que asume la organización política y social al interior del complejo, caracterizada de distintos modos por los autores que abordaron el fenómeno (7). A su vez, esta expansión a nivel económico y social otorgó relevancia a delitos que tendrían lugar al interior de la feria: ocupación ilegal del espacio público, evasión impositiva, precarización y explotación laboral (o "trabajo esclavo", como con frecuencia se lo denomina en la prensa gráfica y otros medios de comunicación) y trata de personas (Gago, 2012; Ossona, 2010a; 2010b; Schiavo et al., 2016) (8).

En relación a los delitos mencionados, parte de la investigación académica en ciencias sociales (Abba, 2009; Albisu, 2011; Campos, 2008; Dewey, 2014; 2015; 2017; Henkel, 2016; Ossona, 2010a; 2010b; 2017; Pogliaghi, 2008; 2010; Sassen, 2011; Savini, 2011), se focalizó en el estudio de La Salada a partir de la distinción entre las dimensiones de formalidad e informalidad (9) (y en muchos casos se hizo particular hincapié en las condiciones laborales de los trabajadores textiles que producen la mercadería ofrecida en la feria, provenientes en su mayoría de Bolivia). Sin embargo, algunos autores han sostenido un enfoque que incorpora otras dimensiones del fenómeno, buscando abordar al complejo comercial a partir de su positividad, esto es, centrándose en las características que asumen las relaciones sociales al interior de la feria.

Considero que una revisión de la literatura académica referente a La Salada nos aportará un panorama orientador acerca de los modos en que diferentes investigaciones han problematizado el fenómeno, así como también de aquellas dimensiones que los respectivos estudios decidieron abordar. Considero, además, que una revisión de este tipo favorecerá la identificación de divergencias y puntos de contacto en los hallazgos producidos por estos trabajos, los cuales pueden ser tomados de base para realizar futuros aportes sobre la temática.

El rastreo bibliográfico se realizó a través del motor de búsqueda de Google Académico utilizando como entrada los términos Feria de La Salada y La Salada. Se seleccionaron aquellas investigaciones que toman al complejo ferial como objeto de estudio. Este proceso de

Question, Vol. 1, N. ${ }^{\circ}$ 63, julio-septiembre 2019. ISSN 1669-6581

Instituto de Investigaciones en Comunicación | Facultad de Periodismo y Comunicación Social | Universidad Nacional de La Plata 
búsqueda fue actualizado en sucesivas oportunidades luego de periodos de cuatro o seis meses. A su vez, el corpus de trabajos fue ampliado mediante la incorporación de bibliografía pertinente referenciada en los trabajos ya seleccionados, y de artículos, trabajos o capítulos de libros sugeridos por especialistas en eventos académicos y durante conversaciones informales. En la búsqueda se identificaron 25 trabajos de investigación publicados entre 2008 y 2017 , entre los que podemos mencionar: dos libros publicados por investigadores en ciencias sociales, cuatro capítulos de libro, una tesis de posgrado, ocho artículos publicados en revistas académicas, dos informes llevados a cabo por entidades estatales (la Administración Federal de Ingresos Públicos y el Ministerio de Trabajo), cuatro ponencias presentadas en congresos nacionales e internacionales y 4 artículos publicados en revistas más cercanas a la divulgación. Se tomó la decisión de incorporar estos últimos trabajos al corpus debido a que en la mayoría de los casos sus autores (o al menos uno de ellos cuando se trató de artículos en coautoría) provienen del ámbito académico en las ciencias sociales, tratándose de investigadores formados (Abba, 2009; Sassen, 2011; Wilkis y Hacher, 2011) o en formación (Henkel, 2016). A su vez, algunos de estos trabajos (Henkel, 2016) discuten con aportes de investigaciones previas o incluso son retomados por estudios de los primeros grupos (Abba, 2009).

A partir de la revisión de algunas de las principales investigaciones referentes a la feria de La Salada, pude observar que uno de los principales criterios de distinción entre estos estudios radica en el modo en que se posicionan ante la existencia misma de la feria. Las investigaciones que focalizan sus análisis a partir de una distinción entre formalidad e informalidad tienden a cuestionar la legitimidad de la existencia del complejo en sí, mientras que en otros estudios se observan posicionamientos que sostienen que un enfoque concentrado únicamente en la precarización laboral o en otro tipo de delitos resulta insuficiente para un abordaje integral del fenómeno. Tomando esto en consideración, podemos organizar a los estudios referentes a la feria de La Salada en dos grupos: a. aquellos trabajos que focalizan sus análisis a partir de una distinción antinómica entre formalidad e informalidad; b. investigaciones que centran el foco de sus análisis en el tipo de relaciones sociales que se producen al interior de la feria y en prácticas sociales que consideran distintivas de este espacio.

En los siguientes apartados se desarrollarán las características generales de las investigaciones de estos grupos destacando las formas en que abordan el fenómeno, sus discrepancias, puntos de contacto y áreas de vacancia sugeridas para posteriores trabajos. 
Investigaciones que abordan al complejo de La Salada a partir de enfoques centrados en la distinción formalidad-informalidad

La mayoría de las investigaciones de este grupo estudian el fenómeno de la feria a partir de una distinción (muchas veces no explícita) entre lo formal y lo informal. Se puede observar que uno de los aspectos más abordados suelen ser las condiciones de precarización y explotación que padecen los trabajadores textiles, en su mayoría migrantes provenientes de Bolivia (10) (Campos 2008; Henkel, 2016; Ossona 2010a; 2010b; 2017; Pogliaghi, 2008; 2010; Sassen, 2011; Savini, 2011). Como características comunes de estas condiciones laborales, los autores mencionan: bajos salarios (el pago suele ser por prenda realizada), contratos laborales no registrados, jornadas de hasta dieciocho horas y ambientes de trabajo insalubres (relacionados con condiciones de hacinamiento, mala calidad de iluminación y exposición durante períodos prolongados de tiempo a ruidos fuertes y al polvo producido por las máquinas).

Otro de los aspectos destacados en estas investigaciones son los modos de reclutamiento de mano de obra. De acuerdo a lo detallado por estos estudios, los trabajadores textiles son reclutados con frecuencia por migrantes de sus propias redes sociales, quienes fomentan expectativas favorables sobre posibilidades de trabajo y progreso que rara vez se cumplen (por lo menos en el plazo inmediato), quedando los ingresantes endeudados desde el inicio con los talleristas que costearon su viaje (Ossona, 2010a; 2010b; Henkel, 2016; Sassen, 2011).

Algunos de estos autores enfatizan su conceptualización de La Salada como consecuencia de las políticas neoliberales de los años noventa (Sassen, 2011; Savini, 2011; Henkel, 2016). Por otro lado, en varios de estos trabajos se señala la funcionalidad que cumple la existencia del complejo ferial con otros sectores más formalizados de la economía. Tanto Henkel (2016) como Sassen (2011) y Campos (2008) destacan que los talleres textiles que producen para La Salada trabajan también para las grandes marcas. Henkel (2016), además, explicita que los trabajadores que se desempeñan en estos talleres resultan funcionales a una política de ajuste, cumpliendo el rol de competidores para trabajadores del mismo rubro que se desempeñan en condiciones de mayor formalidad.

Savini (2011), por su parte, plantea el papel de un Estado informal (informal state) como condición necesaria para la existencia y el desarrollo de La Salada. De acuerdo a esta autora, son las relaciones de connivencia entre la feria y una parte de un "Estado dividido", las que 
permiten la reproducción de la informalidad. En esta misma línea, los trabajos de Dewey (2014; 2015 ; 2017) muestran los vínculos entre las dinámicas de funcionamiento de las ferias y un sector del Estado en su nivel municipal. Según el sociólogo, el Estado permite estas dinámicas (que como anteriormente se mencionó, pueden incluir la ocupación ilegal del espacio público, la falsificación de marcas y el trabajo no registrado) a cambio de ciertos recursos económicos expresados en el pago de determinados tributos. Esta presencia ilegal del Estado, como la denomina Dewey, está orientada también por el interés en sostener los beneficios que supone la contención social llevada a cabo por las ferias (que según el autor se observan en la generación de empleo, si bien precario e informal, para un amplio sector de la población y en el acceso a bienes de indumentaria para personas que, por sus recursos económicos, no los podrían adquirir al interior de circuitos de comercio formales). Es importante destacar que Dewey, además, es uno de los pocos autores que hasta el momento dialogan de manera explícita con otros trabajos que comparten el mismo objeto de estudio, señalando áreas de vacancia. Según el autor, la mayoría de las investigaciones no contextualizan el fenómeno de la feria con el devenir de la industria textil durante las últimas cuatro décadas y esto dificulta "una visión del conjunto que dé cuenta del verdadero perfil como centro comercial" (Dewey, 2017: 202).

Considero significativo aclarar que, al interior de este conjunto de investigaciones, pueden encontrarse matices en los posicionamientos que presentan los autores con respecto a la existencia de la feria. Mientras autores como Dewey (2015; 2017), Henkel (2016), Ossona (2010a; 2010b; 2017), Sassen (2011) y Savini (2011) conciben a La Salada como expresión de una desigualdad social y económica que se profundizó a través de políticas neoliberales de los noventa, Abba (2009) y Campos (2008) muestran posturas más moderadas en este aspecto. Si bien éstos últimos reconocen las condiciones de comercio informal y de precarización y explotación laboral a las que son sometidos los trabajadores textiles, ambos consideran que la expansión a nivel social y económica que tuvo la feria, la oferta de productos accesibles para sectores de bajos recursos económicos, así como también el volumen de puestos de trabajo generados, ameritarían intentos de formalización de este sector de la economía.

Por último, y como punto de contacto con las producciones académicas que analizaré en el siguiente apartado, Campos (2008: 234) señala las limitaciones que una concepción de la informalidad que sólo se reduzca en términos de actividades no registradas en el sistema tributario, poco productivas o de supervivencia podría acarrear: "esa caracterización generalizante, compartida por buena parte del ámbito académico, pierde la posibilidad de captar la heterogeneidad de situaciones existentes dentro de la informalidad". Con respecto al 
caso específico del complejo ferial, el autor argumenta que ese tipo de definición sobre la informalidad es puesta en tensión cuando se aplica a La Salada, ya que en muchos casos, los locales de la feria realizan un pago impositivo bajo la figura del monotributo y no podrían ser consideradas unidades económicas informales en un sentido estricto.

En el siguiente apartado se desarrollarán las características de investigaciones cuyo enfoque sobre la feria se centra en su dimensión productiva, esto es, a partir de las formas que asumen las relaciones y prácticas sociales generadas al interior de este espacio.

Estudios sobre La Salada que se concentran en el tipo de prácticas y relaciones sociales específicas que allí se producen

Al interior de este grupo encontramos investigaciones que estudian el fenómeno centrándose en el tipo relaciones y prácticas sociales que se producen al interior de la feria. Si bien la mayoría de estos trabajos (Benencia y Canevaro, 2017; Forment, 2015; Gago, 2011; 2012; 2014; D'Angiolillo et al., 2010; Wilkis y Hacher, 2012) reconocen de forma explícita las condiciones laborales que se dan en los talleres textiles, existe una intención por parte de sus autores de realizar un abordaje de la feria como un espacio social (Bourdieu, 2000; 2003; 2011), pretendiendo incorporar los puntos de vista de los actores, sus distintas posiciones, trayectorias, interacciones y estrategias de ascenso al interior del complejo. En algunos casos (Benencia y Canevaro, 2017; Gago, 2014; Wilkis y Hacher, 2012) se observa un esfuerzo por incorporar testimonios en primera persona de los feriantes.

Gago (2012) define a su perspectiva como "extra-moral", planteando el objetivo de estudiar el fenómeno de La Salada a partir de su dimensión productiva. En este sentido, resulta interesante el modo en que la autora recupera la noción de informalidad, ya no enmarcada en una distinción entre lo legal y lo ilegal, sino como un "principio de creación de realidad" e incluso como "praxis que busca nuevas formas" (Gago, 2012: 21). Como punto de contacto con las limitaciones señaladas por Campos (2008) acerca de una concepción de la informalidad reducida a una dimensión deficitaria, la autora reelabora este concepto: 
Lo informal en este sentido no refiere a lo que no tiene forma, sino a la dinámica que inventa y promueve nuevas formas (productivas, comerciales, relacionales, etc), poniendo el eje en el momento procesual de producción de nuevas dinámicas sociales (Gago, 2012: 21).

Con distintos niveles de profundidad, y focalizando sus análisis en diferentes dimensiones, los estudios de este grupo abordan múltiples aspectos del complejo ferial, como, por ejemplo, los roles ocupados por los actores (entre los que se encuentran vendedores ambulantes, carreros, trabajadores textiles, talleristas, puesteros y administradores de los predios) y sus respectivas jerarquías (D’Angiolillo et al., 2010; Gago, 2012; 2014), las festividades típicas propias de cada uno de los predios que componen al complejo, su articulación con las identidades nacionales que predominan en cada uno de éstos, los modos de organización y representación política al interior y hacia afuera del complejo (11) (D’Angiolillo et al., 2010; Forment, 2015; Gago, 2012; 2014; Velázquez, 2013), los distintos modos de apropiación y uso de las tecnologías de la información y comunicación (TIC) por parte de feriantes para publicitar sus artículos y al espacio de la feria (Schiavo et al., 2011; 2013; 2014) y experiencias de movilidad social ascendente vivenciadas por algunos de sus participantes (Benencia y Canevaro, 2017).

En referencia a los modos de organización y de trabajo que se encuentran en el complejo ferial, Gago (2012) utiliza el término "capital comunitario" para referirse a éstas lógicas de organización en el caso particular de los migrantes bolivianos. Este capital comunitario, según la autora, funcionaría como recurso de autogestión y movilización, pero al mismo tiempo también de servidumbre, sometimiento y explotación. Esta perspectiva es cuestionada por autores cuyos trabajos se focalizan en las condiciones de explotación laboral al interior de la feria. Henkel (2016: 1) concibe a la perspectiva extra-moral propuesta por Gago como una "ponderación cultural de la informalidad". A su vez, la noción de "capital comunitario" es interpretada por Ossona (2010: 13) como una "explotación endógena de pobres sobre pobres" y por Henkel (2016: 5) como la "explotación salvaje de un compadre" (12).

La mayoría de estas investigaciones presentan una estrategia metodológica cualitativa que incluye entrevistas y observación participante. Si bien esto no es explicitado en todos los casos, es posible inferirlo por la densidad descriptiva con la que se ilustran situaciones cotidianas concretas de la feria y por la cita directa de testimonios de feriantes. Entre las excepciones podemos encontrar los trabajos de Forment (2015), de Schiavo, Rodríguez y Vera (2011; 2013; 2014) y de Schiavo, Vera, y Dos Santos Nogueira (2016), los cuales trabajan 
fundamentalmente con documentos disponibles en sitios web o publicados por la prensa gráfica.

El estudio de Forment (2015) se centra en los modos en que las prácticas de los feriantes producen nuevas formas de organización y autorrepresentación que el autor denomina como "democracia plebeya". En esta línea, considero interesante destacar también los trabajos de Schiavo, Rodríguez y Vera $(2011 ; 2013 ; 2014)$, en los cuales se analizan distintos sitios de internet vinculados a la feria y a partir de allí se observan los diferentes modos en que el espacio de La Salada se expande hacia otros sectores sociales, además de posibilitar diversos tipos de interacciones entre quienes participan de la feria. Por último, el trabajo de Schiavo, Vera y Dos Santos Nogueira (2016), mediante la estrategia de análisis de documentos, aborda las representaciones sociales que se construyen en la prensa gráfica sobre La Salada. Luego de llevar a cabo un análisis de las notas publicadas por los diarios La Nación y Página/12 entre los años 2010 y 2012, las autoras sostienen que los medios gráficos (y los medios de comunicación en general) construyen una imagen de la feria en la cual se resaltan aspectos de la informalidad que los propios medios califican como negativos, peligrosos 0 indeseables (Schiavo et al., 2016), destacando noticias vinculadas a situaciones de violencia o a la existencia de talleres clandestinos y las condiciones laborales que se dan al interior de éstos. $\mathrm{Si}$ bien las autoras señalan que estas representaciones aparecen con mayor frecuencia en uno de los periódicos, concluyen que el análisis de los medios gráficos (aún cuando se trabaje con publicaciones de posicionamientos políticos bien distintos) resulta insuficiente para abordar la complejidad del fenómeno de La Salada e insisten en la necesidad de incorporar otras fuentes de información como, por ejemplo, los actores involucrados. En este sentido, podríamos considerar que la noción de informalidad como concepto que permita abordar las nuevas formas que se producen al interior de la feria (y que no se reduzca únicamente a elementos negativos o deficitarios) es un presupuesto compartido por los trabajos de este grupo.

\section{Algunas reflexiones a modo de cierre}

En este trabajo se buscó trazar un recorrido a través de distintas producciones académicas que tomaron al complejo comercial de La Salada como objeto de estudio. En este proceso se identificó como principal criterio de distinción entre las investigaciones a la elección del eje a partir del cual se deciden centrar los análisis sobre la feria. Si bien es importante destacar que 
toda selección y clasificación de investigaciones es arbitraria, considero que este esquema resulta útil a los fines de visibilizar los principales enfoques y las dimensiones que se tomaron para abordar al complejo de La Salada.

Considero interesante destacar que en estos trabajos no se encontraron grandes discrepancias o contradicciones en los hallazgos o en los aspectos de la feria que se exponen. Si bien estos dos grupos de investigaciones sostienen posturas distintas con respecto al complejo ferial, los hechos que ilustran, independientemente de la valoración que se les atribuye, suelen coincidir o complementarse.

La vigencia de una informalidad económica (expresada principal, pero no exclusivamente, bajo la forma del empleo no registrado) y la presencia de migrantes bolivianos, que trabajan en condiciones precarias y sin ningún tipo de regulación legal son reconocidas por autores de ambos grupos de investigaciones. No obstante, estos estudios se diferencian en la forma en que interpretan estos hechos concretos. Mientras Gago (2012) sostiene la existencia de un "capital comunitario" para describir los modos en que estos feriantes se organizan con el fin de impulsar sus emprendimientos y cubrir distintas necesidades de su comunidad, autores como Ossona (2010a; 2010b) o Henkel (2016) no rechazan en sentido estricto la existencia de estos lazos, pero sí les atribuyen otro tipo de valoración. Donde algunos autores observan formas de organización y cooperación, otros perciben la explotación al interior de una misma comunidad nacional. Considero que estas discrepancias en las formas de conceptualizar un mismo fenómeno responden a preocupaciones diferentes de quienes realizan estas investigaciones.

Por lo menos hasta el momento de elaboración de este artículo, puede observarse que los estudios que se han propuesto realizar un abordaje de la feria centrándose en aquello nuevo que se produce allí, han permitido alumbrar aristas que otras investigaciones no suelen contemplar. Entre los aspectos abordados por estos enfoques podemos encontrar, por ejemplo, la representación política de la feria "hacia afuera", los modos en que se expresan las identidades nacionales en cada predio, las trayectorias de feriantes, historias de movilidad social ascendente, y los posicionamientos y puntos de vista de los feriantes sobre estos aspectos.

Este tipo de enfoque, si bien puede ser considerado por otros autores como "meramente culturalista" (Henkel, 2016: 9), permite abordar nuevos aspectos del fenómeno. En este sentido, considero que la noción de informalidad que proponen autoras como Gago (2012), Schiavo, Vera y Dos Santos Nogueira (2016) o incluso autores como Campos (2008), resulta provechosa para identificar nuevas dimensiones y problemáticas referidas a La Salada.

Question, Vol. 1, N. ${ }^{\circ}$ 63, julio-septiembre 2019. ISSN 1669-6581

Instituto de Investigaciones en Comunicación | Facultad de Periodismo y Comunicación Social | Universidad Nacional de La Plata 
Si bien su principal preocupación es el trabajo informal, autores como Campos (2008) o Albisu (2011) señalan en sus respectivos estudios que, en etapas posteriores de su expansión, la feria comenzó a captar visitantes de los sectores medios. Por otro lado, Schiavo, Vera, y Dos Santos Nogueira (2016) señalan la valoración positiva que diversos públicos del país y del extranjero hacen de los productos textiles de La Salada. Estas autoras destacan el interés en abordar las percepciones que tienen sobre la feria estos nuevos públicos que en determinados casos disponen de una capacidad de consumo que hace electiva su visita al complejo ferial. Esta propuesta resulta de sumo interés, si se considera que es un aspecto apenas mencionado por algunos autores e implica una forma de abordar manifestaciones de los niveles más recientes que alcanzó la expansión de la feria. En un sentido similar, y si bien centran sus enfoques en una distinción entre formalidad e informalidad, autores como Pogliaghi (2008; 2010), Albisu (2011) y Dewey $(2015 ; 2017)$ explicitan que por lo menos la mitad de las prendas que se comercializan en La Salada no son falsificaciones de marcas, lo cual nos sugiere el interés por abordar con mayor profundidad el surgimiento y posible desarrollo de estos emprendimientos.

Los estudios mencionados brindan indicios para profundizar (o por lo menos atender con un detenimiento mayor) durante futuros trabajos. Ya se trate de estudios focalizados en las condiciones de informalidad en las que opera la feria (informalidad entendida como funcionamiento por fuera de la regulación estatal, que, según varios de estos mismos trabajos, dista de ser total o absoluta) o en aquellas relaciones y prácticas sociales originadas a partir del espacio de La Salada, la mayoría de estos trabajos alumbran aristas que ameritan un abordaje más profundo. Aspectos como la proporción de comercios dentro del complejo que ofrecen indumentaria que no infringe la ley de marcas (sea esto porque no la tienen o porque tienen marcas propias), los relativos niveles de formalización que presentan algunos de estos locales y el acercamiento a estos circuitos por parte de consumidores provenientes de los sectores medios (práctica que en la prensa gráfica y de modo excepcional se describe como "consumo inteligente") (13) da cuenta de un potencial creativo y de expansión que motiva futuros abordajes. Para esto considero fundamental continuar con la utilización de técnicas de investigación cualitativa, como la observación participante durante los días en que funcionan las ferias y las entrevistas en profundidad a sus principales protagonistas, no sólo a fabricantes y comerciantes (roles que en la práctica suelen ser desempeñados por las mismas personas), sino también ( $y$ en particular) a aquellos que, por diversos motivos, eligen adquirir estos artículos. 
Notas

(1) Una versión preliminar de este trabajo (Bello, 2017) fue presentada en las IX Jornadas de Jóvenes Investigadores del Instituto de Investigaciones Gino Germani el 6 de noviembre de 2017. Para la presente versión fueron incorporadas quince investigaciones al corpus analizado, se reelaboraron algunas definiciones sobre el modo de comprender a los dos conjuntos de estudios, se explicitaron aspectos vinculados a los criterios de rastreo y selección de estos trabajos y se profundizaron algunos hallazgos.

(2) Quiero expresar mi agradecimiento a la Dra. Guillermina Comas y a la Lic. Lucía Cavalo por sus comentarios a versiones anteriores de este artículo. También quiero agradecer a la Dra. Leticia Pogliaghi, a la Dra. Esther Schiavo y a la Dra. Paula Vera por haberme facilitado trabajos de su autoría que, desde diferentes enfoques, abordaron el fenómeno de La Salada.

(3) Campos (2008) y Pogliaghi $(2008 ; 2010)$ enfatizan este aspecto para destacar la capacidad de adaptación que tuvo la feria a la hora de comercializar tanto importaciones como producciones de fabricación local.

(4) Si bien en la actualidad siguen existiendo puestos de venta ubicados en la vía pública, éstos distan de constituir las dimensiones que tenía la feria de La Ribera, que abarcaba entre diez y quince cuadras a la vera del Riachuelo, empleando cerca de 7000 puestos.

(5) Los primeros dos lugares fueron ocupados por Unicenter y Abasto Shopping respectivamente (Abba, 2009).

(6) Estos números corresponden a estudios que datan de 2012. El actual contexto de recesión económica, aumento de los costos de las materias primas y de los servicios básicos y la caída en el consumo ameritan futuras consultas acerca de la vigencia de estas cifras.

(7) Ossona (2010a: 27) describe al funcionamiento de la feria como "un Estado dentro del Estado". Gago (2012) concibe a la existencia de La Salada como una expresión "desde abajo" de las políticas neoliberales, mientras que D'Angiolillo et al. (2010: 23) la define como una "centralidad periférica suburbana". Por su parte, Forment (2015) utiliza el concepto de "democracia plebeya" (plebeian democracy) para caracterizar los modos en que quienes participan en el complejo ferial organizan sus prácticas.

Question, Vol. 1, N. ${ }^{\circ}$ 63, julio-septiembre 2019. ISSN 1669-6581

Instituto de Investigaciones en Comunicación | Facultad de Periodismo y Comunicación Social | Universidad Nacional de La Plata 
(8) Entre las noticias más relevantes publicadas durante los últimos años sobre La Salada encontramos a la detención de Jorge Castillo y Enrique 'Quique' Antequera, administradores de los predios de Punta Mogote y Urkupiña respectivamente, por los delitos de asociación ilícita. Véase: “Detuvieron al 'Rey de La Salada', 21/06/2017, La Nación y "Arrestaron al otro hombre fuerte de La Salada", 11/08/2017, Página/12.

(9) Incluyo a los enfoques que abordan a las ferias a partir de una distinción entre legalidad e ilegalidad (Dewey, 2014; 2015; 2017) como casos específicos dentro de este grupo.

(10) Si bien en estos estudios se menciona también la presencia de actores de otras nacionalidades como argentinos, paraguayos, peruanos y coreanos, el trabajo textil suele vincularse principalmente a los migrantes bolivianos.

(11) Considero significativo destacar que varios de estos aspectos fueron abordados también por Pogliaghi (2008; 2010) y por Ossona (2010). Sin embargo, sus enfoques y posicionamientos se orientan a priorizar las condiciones de informalidad laboral como problema de investigación central en sus trabajos.

(12) Con respecto a esta cuestión considero que el trabajo de Campos vuelve a ubicarse en un punto de enlace entre ambos grupos. Si bien el eje de su trabajo se centra en la problemática del trabajo informal (y en el marco de su informe éste no se reduce únicamente al trabajo textil para La Salada), Campos (2008) describe al espacio de la feria como un espacio de competencia entre feriantes pero a la vez también como un espacio de cooperación en el que circula información sobre tendencias de moda que resulta provechoso para el funcionamiento del mercado.

(13) Véase al respecto "Los ricos también cuidan su bolsillo", Alfredo Sáinz, Diario La Nación, 29/11/2009.

\section{Bibliografía}

Abba, A. P. (2009). La Salada. Una mega centralidad informal en la periferia metropolitana de Buenos Aires. Revista digital "Café de las Ciudades", 8(86). Recuperado de http://www.cafedelasciudades.com.ar/economia_86.htm 
Juan Martín Bello. Un polo textil impulsado desde la periferia. Abordajes sobre La Salada a partir de investigaciones en Ciencias Sociales

Albisu, C. E. (2011). El fenómeno de las ferias ilegales como foco de informalidad laboral: el caso de La Salada. Cuadernos del Instituto AFIP C25. Recuperado de https://www.afip.gob.ar/educacionTributaria/BibliotecaDigital/documentos/C25.pdf

Bello, J. (2017). Nuevas formas de organización económica y social: conceptualizaciones de la Feria de La Salada a partir de investigaciones académicas. Ponencia presentada en IX Jornadas de Jóvenes Investigadores del Instituto de Investigaciones Gino Germani, Ciudad de Buenos Aires.

Benencia, R. y Canevaro, S. (2017). Migración boliviana y negocios. De la discriminación a la aceptación. La Salada como fenómeno social. REMHU-Revista Interdisciplinar da Mobilidade Humana, 25(49).

Bourdieu, P. (2000). La distinción: criterios y bases sociales del gusto. Madrid: Taurus.

Bourdieu, P. (2003). Creencia artística y bienes simbólicos: elementos para una sociología de la cultura. Buenos Aires: Aurelia Rivera.

Bourdieu, P. (2011). Cuestiones de Sociología. Madrid: Akal.

Campos, M. (2008). La heterogeneidad del trabajo informal. Los resultados de un estudio cualitativo sobre los sectores del comercio textil, la construcción y el transporte. En Banco Mundial-MTEySS. Aportes a una nueva visión de la informalidad laboral en la Argentina (pp. 231-238). Recuperado de https://bit.ly/2kiujGu

D’Angiolillo, J.; Dimenstein, M.; Di Peco, M.; Guerin, A.; Massida, A.; Molins, M. C.; Muñoa, N.; Scarfi, J. P. y Torroja, P. (2010). Feria La Salada: una centralidad periférica intermitente en el Gran Buenos. En Gutman, M. (coord.). Argentina: persistencia y diversificación, contrastes e imaginarios en las centralidades urbanas (pp. 182-83). Quito, Ecuador: Olacchi.

Dewey, M. (2014). Taxing the shadow: The political economy of sweatshops in La Salada, Argentina. MPIfG Discussion Paper, 14/18, Max-Planck-Institut für Gesellschaftsforschung, Köln.

Dewey, M. (2015). El orden clandestino: Política, fuerzas de seguridad y mercados ilegales en la Argentina. Buenos Aires: Katz Editores.

Dewey, M. (2017). La importancia de La Salada. Protección gubernamental y expansión del mercado informal de la indumentaria. En Zarazaga, R. y Ronconi, L. (Comp.) Conurbano infinito: Actores políticos y sociales, entre la presencia estatal y la ilegalidad (pp. 177-205). Buenos Aires: Siglo XXI.

Forment, C. (2015). Ordinary Ethics and the Emergence of Plebeian Democracy across the Global South. Buenos Aires's La Salada Market. En Current Antropology, 56(S11). 
Juan Martín Bello. Un polo textil impulsado desde la periferia. Abordajes sobre La Salada a partir de investigaciones en Ciencias Sociales

Gago, V. (2011). Entre la microeconomía proletaria y la red transnacional: la feria popular como desafío a la ciudad neoliberal. La Biblioteca, 11. Buenos Aires, pp. 238-255.

Gago, V. (2012). La Salada: ¿un caso de globalización «desde abajo»? Territorio de una nueva economía política transnacional. Nueva Sociedad, 241, pp. 63-78.

Gago, V. (2014). La razón neoliberal. Economías barrocas y pragmática popular. Buenos Aires: Tinta Limón.

Henkel (2016). La 'cultura' de La Salada: inmigración y trabajo esclavo. Revista Luna Roja, Crisis y Cultura, 3.

Ossona, J. L. (2010a). El shopping de los pobres: Anatomía y fisiología socioeconómica y política de La Salada. Documento presentado en VI Congreso del CEISAL Independencias-Dependencias-Interdependencias en la Univ. de Toulouse-Le Mirail, Francia.

Ossona, J. L. (2010b). La inmigracion paraguaya y boliviana en el norte de Lomas de Zamora durante los últimos veinte años. Documento presentado en VI Congreso del CEISAL "Independencias-Dependencias-Interdependencias" en la Univ. de Toulouse-Le Mirail, Francia.

Ossona, J. L. (2017). Detrás de La Salada. Ocupaciones territoriales y economías clandestinas en la localidad de Santa Catalina, Lomas de Zamora. En Zarazaga, R. y Ronconi, L. (Comp.) Conurbano infinito: Actores políticos y sociales, entre la presencia estatal y la ilegalidad (pp. 207-240). Buenos Aires: Siglo XXI.

Pogliaghi, L. (2008). Informalidad urbana. Una aproximación a partir de un estudio de caso: las ferias de La Salada. Lomas de Zamora (2006-2007). Universidad Nacional de General San Martín, Buenos Aires, mimeo.

Pogliaghi, L. (2010). El trabajo en el comercio de indumentaria en ferias: una aproximación a partir de un estudio de caso en el conglomerado de ferias de La Salada. En Palomino, H. (Comp.). La nueva dinámica de las relaciones laborales en la Argentina (pp. 67-95). Buenos Aires: Jorge Baudino Ediciones.

Sassen, S. (2011). La Salada: The Largest Informal Market In South America. Forbes. Recuperado de https://bit.ly/2kiqUaG

Savini, R. (2011). Enduring informality: the case of La Salada market and the informal state in Argentina. MSc in Development Management, London School of Economics and Political Science.

Schiavo, E.; Rodríguez, S.; Vera, P. y C. Dos Santos Nogueira (2011). Vínculos entre informalidad e inclusión social en metrópolis latinoamericanas. Caso Feria La Salada. 
XXVIII Congreso Internacional de Asociación Latino- Americana de Sociología ALAS, Recife, Brasil.

Schiavo, E.; Rodríguez, S. y Vera, P. (2013). Appropriation of ICTs by informal communities in metropolitan cities. The case of the "La Salada" market in the Latin American context. The Journal of Community Informatics (JOCl), 3(9).

Schiavo, E.; Rodríguez, S. y Vera, P. (2014). Apropiación de TIC por parte de comunidades informales en ciudades metropolitanas en el contexto latinoamericano. El caso de la feria 'La Salada'. En Finquelevich, S. (Coomp.). Innovación abierta en la sociedad de conocimiento: redes transnacionales y comunidades locales. Buenos Aires: Instituto de Investigaciones Gino Germani.

Schiavo, E.; Vera, P. y Nogueira, C. D. S. (2016). La Salada: imaginarios y representaciones de la informalidad y las desigualdades territoriales en la prensa escrita. Questión, 1(50), pp. 387-404.

Velázquez, M. A. (2013). El derecho a la movilidad urbana en la Región Metropolitana de Buenos Aires y sus conflictos jurisdiccionales: su emergencia en el corredor sudoeste con el caso de la feria de La Salada. Ponencia presentada en VII Jornadas Santiago Wallace de Investigación en Antropología Social, Sección de Antropología Social. Instituto de Ciencias Antropológicas, Facultad de Filosofía y Letras, UBA, Buenos Aires.

Wilkis, A., y Hacher, S. (2012). La China invisible. Revista Anfibia, Universidad Nacional de San Martín. Recuperado de http://www.revistaanfibia.com/cronica/la-china-invisible/

\section{Notas periodísticas}

La Nación (29/11/2009). Los ricos también cuidan su bolsillo. Recuperado de https://www.lanacion.com.ar/economia/los-ricos-tambien-cuidan-el-bolsillo-nid1205468

La Nación (21/06/2017). Detuvieron al 'Rey de La Salada'. Recuperado de https://www.lanacion.com.ar/seguridad/detuvieron-al-rey-de-la-salada-se-resistio-a-los-tiros-ehirio-a-un-policia-nid2035591

Diario Página/12 (11/08/2017). Arrestaron al otro hombre fuerte de La Salada. Recuperado de https://www.pagina12.com.ar/55939-una-detencion-para-cerrar-la-campana 